\title{
Smart Water Tank Monitoring and Booking Using IoT Framework
}

\author{
Esraa Khalfan Mubarak Al \\ Wadhahi \\ Syed Ateeq Ahmed
}

\author{
Middle East College \\ Middle East College
}

\begin{abstract}
Water is at the heart of sustainable development, which is essential for economic and social development, productive energy for ecological safety and human survival. Water is also a human right. As the world's population increases, the need for a balance between all commercial water resource requirements is needed to allow communities to get enough water. At present, conventional water tanks can neither monitor nor control the water level in tank, leading to large amount of water waste. Also some families often face problems to examine the availability of water in water tanks, including the lack of water supplies in the area because of separation of water supply from the government in new and undeveloped areas. The residents of under developed areas must find a person to fill the tank with water as and when the water is finished. The need of removal of these problems and providing an efficient and economical solution has been the main focus of this research paper. The research paper provides a framework for design and propose a framework of a smart water tank which can be monitored as well as controlled with android application. By using this application (the smart water tank), people can easily place an order using the smart application which notifies the water supplier to refill the water preventing shortage of water.
\end{abstract}

\section{Introduction}

Water is a key requirement of sustainable development, and is essential for economic and social development, productive energy for ecological safety and human survival. With the continuous growth in the human population, water resource requirements are a must in a day to day of life of human beings. In the newly developed areas, as the water supply may not available, inhabitants may face the problem of shortage of water.

In the present era, water is precious and a limited resource and it is crucial for agriculture and industry sector as well as for the survival of human beings and other living creatures on earth.

In today's world, one of the emerging technologies is the Internet of things (IoT). In this technology, millions and millions of devices can communicate with each other, sensing and sharing of information is among the core aspects of this technology. These devices are either connected through an internet protocol which is either private or public (Kulkarni \& Joshi, 2016).

One of the advancements in the internet technology is the internet of things. IoT devices collect, monitor, evaluate and notify the user with the information. This technology is rapidly changing the life of humans into a new level. With the advancements in this technology and using various devices human life is changing from a normal life to a smarter life. Some of the examples as advantages of the emerging technology are smart homes, smart cities, improved healthcare systems. Water monitoring system is also an example of the Internet of Things. IOT technology can be used to monitor water levels and sensors can be applied to obtain information of the water level. Readings from sensor can be sent to the users (Jeughale et al, 2018).

\section{Research Methodology}


The methodology adopted for doing this research is indicated below (Figure 1).

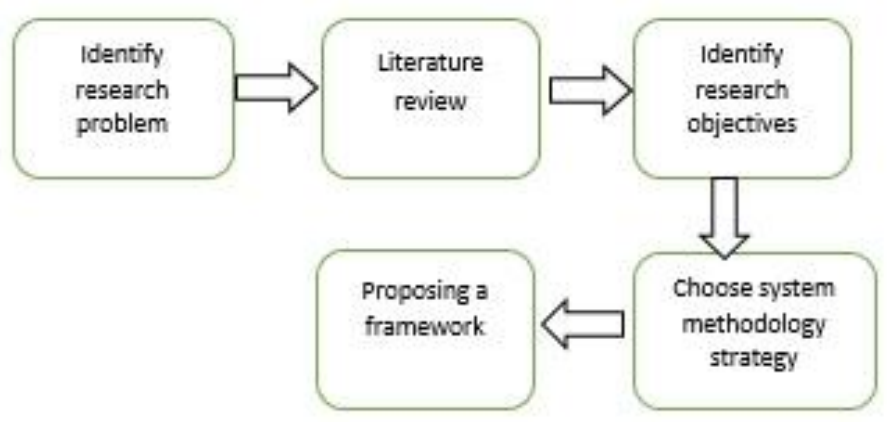

Figure 1. Research Methodology

\section{Literature Review}

A system using the microcontroller with model Atmeag128A is used in managing smart water tank for reduces water wastage and well monitory (Patil \& Singh, 2014). In the research, a model is proposed using two tanks and a reservoir. Each of the water tanks are having indicators. Sensors are using to measure the water level. There is a facility to know the current status of water level in the tank on an LCD display. Both the water pumps are attached with the tank. Once the tank is empty, the pump will fill the tank by taking the water from the reservoir. Water levels are identified in the tank as low, middle and high level. The higher level is used to switch off the pump. This system to manage water level, displays the current level of water in the tank. Sensors are installed to measure the water level.

A new revolution in internet which is internet of things, mixture of communication and technology using a various area of application. It's not what we know about only electronic things or device or the products of high technology such as vehicles and tools but things that we don't think about it like food, clothing, animal, chair, and water....etc. with internet of things the communication is extended to all of thing surround us. And this technology provides solutions based on the integration of information. So internet of thing is not a single of technology but it's a mixture of technology.

Internets of thing carry some characteristic such as interconnectivity with it anything can connect with information and communication. Secondly, safety designs it for human and includes the safety of our personal. Lastly connectivity been enables to network for accessing for consume and produce data. (Patel \& Patel, 2016).

ESP 8266 is a Wi-Fi based module uses the internet connectivity through the hotspot using proper authentication. As per the requirements it can be customized through code. The ultrasonic sensor can determine the level of water surface and return it to the ESP device. Using internet, ESP can send data to a database in the cloud environment. It can also communicate with the android application. Motor works on the level of water which can be at either maximum or minimum levels.

An ultrasonic sensor can be used which works on ultrasonic sound waves when hit the water surface. In this case, sensor has an attached speaker, is not in contact with the water surface, due to which the life of sensor will be more (Theja, 2018).

For IT projects, relevant system development methodology must be chosen. To choose a methodology, various existing development approaches need to be studied and chose the methodology which is relevant for the proposed system development process. Developing a software system involves various techniques adopted by the IT industry. Various software 
development methodologies need to be compared based on the strengths and weaknesses of the methodologies. Waterfall methodology is linear, where the project owner gets the feedback only at the end. In the prototype methodology, prototypes need to be developed which are time consuming, such a methodology is useful more in case of innovative projects or similar projects were previously not available. Iterative methodology is suitable for medium and large size projects. Rapid application development methodology has less focus on planning related aspects. Dynamic systems development methodology serves the needs as per the requirements. It's a iterative model and time bound (Despa, 2014).

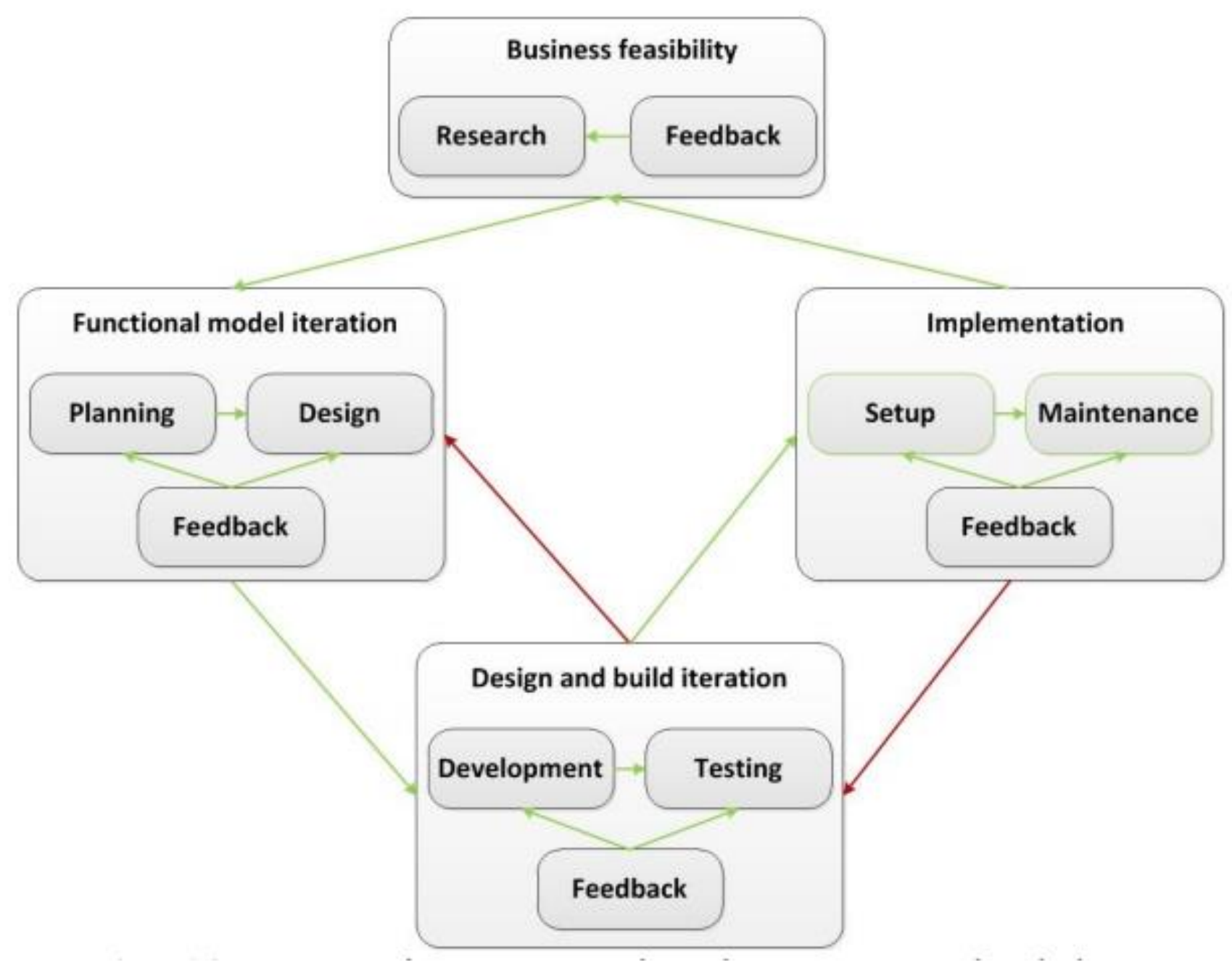

Figure 2. Dynamic systems development methodology (Despa, 2014)

Smartphone is an important innovation of the recent times and it has significant impact on the lifestyle of the current era. It is among the popular operating systems used today. Android operating system, an open source software, freely accessible, however, possesses security challenge. The challenge in mobile application development using android operating system is to be able to use on devices with different configurations, issues with emulators, memory issues for data intensive applications, lack of software and hardware integration. Since android is an open ended software, subjected to risk of malware applications (Kathuria \& Gupta, 2015).

\section{Research Question and Objectives}

This research is focused on integrating the sensors used in water tank with a mobile application, get notification on the predefined levels of water in the water tank and facilitate the water supply 
by order booking through the application.

The related work done indicates some work has been done in this problem area. The work done is partial, the research focuses on providing a complete framework for getting notified of water levels and ease of booking water through the suppliers registered in the application.

\section{Data Collection Techniques and Analysis}

Response is collected from various users using questionnaires. Some interviews were also conducted. The collected data is summarized and analysed.

Figure 3. Data collected on ease of using a mobile application.

Data is collected to know whether an online application on mobile phone will be useful for ease of notifying of water levels in the tank and order supply.

Figure 4. Data collected on usefulness of android application with reference to the research problem area.

Users are also asked with to know the significance of alerting a user in notifying that water level has reached the predefined minimum level so that an order can be booked from the water suppliers (Figure 5).

Figure 5. Response of users indicating the significance of alert to notify the water level.

\section{System Development Methodology for the Proposed Framework}

After comparing various methodologies in the literature review phase, the chosen system development methodology is dynamic systems development methodology. It is an iterative development process, time bound and deadlines need to be strictly followed. In this methodology testing is early done and it is a continuous process. This methodology provides complete documentation and users are actively involved.

\section{Proposed Framework and Application Design}

The proposed framework provides a solution for monitoring water levels in the tank and provides ease of order booking through the application. It is based on IoT framework wherein devices need to communicate.

\section{System Architecture of the Smart Water Tank and Order Booking Application}

System architecture is given as a basic block diagram. Using the smart application in the mobile phone, user can see the water level based the readings send by the ultrasonic sensor fixed in the water tank. The sensor which is fixed in the water tank, detects the water level and sends the notification (Figure 6). 
Figure 6. Block diagram of smart water tank and order booking.

\section{Functional Aspects of the Proposed Framework}

- Sensor: An ultrasonic sensor to measure the water level inside the tank which includes NodeMCU having Wi-Fi.

- Users: Users should be registered in the application giving details including user name and password.

- Water Suppliers: Water suppliers should be registered in the application with proper authentication details and contact numbers.

- Order Booking: Application having an interface to place orders, including choosing suppliers from the available list of suppliers in the application. Once an order is placed water suppliers will get a notification, they can login to the application to see more details of the users who placed the order.

- Water Supply: Water suppliers supply the water as per the order details.

Class diagram of the proposed framework is given below (Figure 7).

Figure 7. Class diagram of the smart water tank application

\section{Research Result and Solution Framework}

The result of this research is a framework where a user can login and check the water level with ease from anywhere. Based on the requirement of water an order booking can be easily done using the application by choosing a water supplier.

The proposed framework provides an easy detection of water level and order booking of water as indicated in the following steps.

Step-1: User has to login using username and password. After connecting with the sensor, checks the water level information as received from the sensor attached in the tank.

Step-2: If user finds that the water level is below minimum required water level, chooses a supplier from the list of suppliers registered in the application and places an order for water supplier.

Step-3: Water supplier will get a notification on his mobile device, log in to the application and gets details of the customer and supplies the water.

\section{Conclusion and Future Work}

Water is a key requirement for the survival of human beings. In this research, a framework is proposed to provide an integrated application to detect the water level and order booking.

This research can be further extended to provide an integrated environment which also detects water leaks, overflow of water thereby saving water and providing a sustainable environment.

\section{References}




\section{Journal of Student Research}

Fourth Middle East College Student Research Conference, Muscat, Sultanate of Oman

Kulkarni, P.V., and Joshi, M.S. (2016) "An IoT based Water Supply Monitoring and Controlling System with Theft Identification" International Journal of Innovative Research in Science, Engineering and Technology, 5(9): 16152-16157

Jeughale,S., Jadhao,A.P., Bachate, P.A., Chankhore, D.A., and Narkhede,V.P. (2018), "Water Tank Level Monitoring using IoT: A Implementation" International Journal of Advent Research in Computer and Electronics (IJARCE).

Patil,Y., and Singh, R. (2014) "Smart Water Tank Management System for Residential Colonies Using Atmega128A Microcontroller" International Journal of Scientific \& Engineering Research, 5(6): 355-357.

Likun, H., Guangping, L and Wenqin, H (2010) "Level Control System of Double-Hold Water Tank Based on Inverse System Method and PID" International Conference on Intelligent Human-Machine Systems and Cybernetics 10(2):129-132

Jiang, W. (2010) "Intelligent Building Control of Water Tank Based on Fuzzy Theory" International Conference on Intelligent Computation Technology and Automation, 2: 549 - 552.

Patel, K.K., and Patel, S.M. (2016) "Internet of Things-IOT: Definition, Characteristics, Architecture, Enabling Technologies, Application \& Future Challenges" International Journal of Engineering Science and Computing, 6(5): 6122-6131

Theja,B. (2018) “IOT based Smart Water Tank with Android

Application" International Journal for Research in Applied Science \& Engineering Technology, 6(1): 2622-2627.

Despa, M.L. (2014) “Comparative study on software development methodologies” Database Systems Journal, 5: 37-56.

Kathuria, A., and Gupta, A. (2015) "Challenges in Android Application Development: A Case Study" International Journal of Computer Science and Mobile Computing, 4(5): 294-299. 